\title{
Toward the Approximate Solution for Fractional Order Nonlinear Mixed Derivative and Nonlocal Boundary Value Problems
}

\author{
Hammad Khalil, ${ }^{1}$ Mohammed Al-Smadi, ${ }^{2}$ Khaled Moaddy, ${ }^{3}$ \\ Rahmat Ali Khan, ${ }^{4}$ and Ishak Hashim ${ }^{5,6}$ \\ ${ }^{1}$ Department of Mathematics, The University of Poonch Rawalakot, Azad Jammu and Kashmir, Pakistan \\ ${ }^{2}$ Department of Applied Science, Ajloun College, Al-Balqa Applied University, Ajloun 26816, Jordan \\ ${ }^{3}$ Department of Mathematics, Faculty of Science and Arts, Shaqra University, Shaqra 11691, Saudi Arabia \\ ${ }^{4}$ Department of Mathematics, University of Malakand, Chakdara, Lower Dir, Khyber Pakhtunkhwa, Pakistan \\ ${ }^{5}$ School of Mathematical Sciences, Universiti Kebangsaan Malaysia, 43600 Bangi, Selangor, Malaysia \\ ${ }^{6}$ Research Institute, Center for Modeling and Computer Simulation, King Fahd University of Petroleum and Minerals, \\ Dharan 31261, Saudi Arabia \\ Correspondence should be addressed to Hammad Khalil; hammadk310@gmail.com
}

Received 13 May 2016; Accepted 19 July 2016

Academic Editor: Douglas R. Anderson

Copyright (C) 2016 Hammad Khalil et al. This is an open access article distributed under the Creative Commons Attribution License, which permits unrestricted use, distribution, and reproduction in any medium, provided the original work is properly cited.

\begin{abstract}
The paper is devoted to the study of operational matrix method for approximating solution for nonlinear coupled system fractional differential equations. The main aim of this paper is to approximate solution for the problem under two different types of boundary conditions, $\widehat{m}$-point nonlocal boundary conditions and mixed derivative boundary conditions. We develop some new operational matrices. These matrices are used along with some previously derived results to convert the problem under consideration into a system of easily solvable matrix equations. The convergence of the developed scheme is studied analytically and is conformed by solving some test problems.
\end{abstract}

\section{Introduction}

Fractional calculus is generalization of integer order integration and differentiation to its noninteger (fractional) order counterpart. Fractional calculus has proved to be a valuable tool in modeling many natural phenomena of physics, chemistry, engineering, aerodynamics, electrodynamics of complex medium, polymer rheology, and so forth [1]. It is well known that fractional order operator is a nonlocal operator. Unlike integer order models the fractional order models have the property that the current state of the system depends on all its historic states. This makes fractional models interesting and remains as an active and hot area of research.

Differential equations are used to model different physical and engineering phenomena. The aim is to know about the future state of the system under consideration. It is some time necessary to model differential equations with different kinds of initial and boundary conditions (depends on the nature of the problem). Various types of conditions which are used as boundary conditions are multipoint local boundary conditions, integral type boundary conditions, multipoint nonlocal boundary conditions, and mixed derivative boundary conditions. The multipoint boundary value problems appear in wave propagation and in elastic stability. For example, the vibrations of a guy wire of a uniform cross section composed of $m$ sections of different densities can be molded as a multipoint boundary value problem (see $[2,3]$ and the references therein).

In this paper, we are interested in finding approximate solutions for the following generalized class of nonlinear coupled systems of fractional order differential equations:

$$
\begin{aligned}
& u^{\sigma_{1}}=f\left(t, u^{\gamma_{1}}, v^{\gamma_{1}}, u, v\right), \\
& v^{\sigma_{2}}=g\left(t, u^{\gamma_{1}}, v^{\gamma_{1}}, u, v\right),
\end{aligned}
$$


where $u=u(t)$ and $v=v(t)$ are the unknown solutions to be determined. These solutions are defined on finite time domain; that is, $t \in[0, \tau]$. The superscripts represent the order of derivatives defined in Caputo sense and are defined as $1<\sigma_{1}, \sigma_{2} \leq 2$, and $0<\gamma_{1} \leq 1 . f$ and $g$ are nonlinear functional of $u$ and $v$ and their fractional derivatives. The method developed in this paper is designed for approximating $u$ and $v$ under any of the following two types of boundary conditions.

(i) M-point nonlocal boundary conditions are as follows:

$$
\begin{aligned}
& u(0)=u_{0}, \\
& u(\tau)=\sum_{i=1}^{m-2} \zeta_{i} u\left(\xi_{i}\right), \\
& v(0)=v_{0}, \\
& v(\tau)=\sum_{i=1}^{m-2} \rho_{i} v\left(\xi_{i}\right),
\end{aligned}
$$

where $u_{0}$ and $v_{0}$ are some real constants and $\xi_{i}$ are intermediate points of the domain defined as

$$
0<\xi_{1}<\xi_{2} \cdots<\xi_{m-2}<\tau \text {. }
$$

(ii) Mixed derivative boundary conditions are as follows:

$$
\begin{aligned}
& u^{\prime}(0)=a_{1} u(0)+a_{2} u(\tau), \\
& u^{\prime}(\tau)=b_{1} u(0)+b_{2} u(\tau), \\
& v^{\prime}(0)=c_{1} v(0)+c_{2} v(\tau), \\
& v^{\prime}(\tau)=d_{1} v(0)+d_{2} v(\tau),
\end{aligned}
$$

where $a_{i}, b_{i}, c_{i}$, and $d_{i}$ are some real constants.

We develop new operational matrices based on Jacobi polynomials and use these operational matrices to develop new formulation for finding approximate solutions for the problems. The technique basically lies in the domain of spectral methods. Spectral methods are widely used for solutions to differential equations, functions approximations, and variational problems. These methods demand approximation of solutions for a problem by truncated series of smooth global functions and provide very accurate approximations for a smooth solution with relatively few degrees of freedom. The main reason behind this accuracy is the behavior of spectral coefficients $a_{n}$, which tend to zero faster than any algebraic power of their index $n$.

Among others, some of the well known mathematicians who successfully applied spectral method are Dehghan [4-9], Bhrawy [10, 11], Doha et al. [12], and Saadatmandi [7, 13, 14]. In these papers the authors solved many scientific problems using spectral methods. Also Dehghan and Shakeri [8] used a seminumerical technique for solving the multipoint boundary value problems. In $[4,6]$ an efficient way is developed for the construction of operational matrices. In [5] the authors used operational matrices of Bernstein polynomials to solve nonlinear age-structured model. We are motivated by the work of Bhrawy and Al-Shomrani [11], who solved fractional order differential equations (both linear and nonlinear) with $m$-point boundary conditions (local) via shifted Legendre polynomials and collocation technique.

To solve the nonlinear coupled system of boundary value problem we implement operational matrix method combined with quasilinearization method. Quasilinearization method was first introduced by Bellman and Kalaba [15] to solve nonlinear ordinary or partial differential equations as a generalization of the Newton-Raphson method. The origin of this method lies in the theory of dynamic programming. In this method, the nonlinear equations are expressed as a sequence of linear equations and these equations are solved recursively. The main advantage of this method is that it converges monotonically and quadratically to the exact solution to the original equations [16]. Also some other interesting works in which qasilinearization method is used for scientific problems are available in [17-19].

In our previous work, we have constructed some efficient methods for the numerical simulations of couple systems of linear fractional differential equations and fractional order partial differential equations [20-22]. Local and nonlocal boundary value problems can be found in [23-25]. For the readers who are new to the field, we recommend studying our previous work in order to get a better understanding.

\section{Preliminaries}

In this section, we recall some basic definitions and known results from fractional calculus. More details can be found in [1].

Definition 1. Given an interval $[a, b] \subset \mathbb{R}$, the RiemannLiouville fractional order integral of order $\alpha \in \mathbb{R}_{+}$of a function $\phi \in\left(L^{1}[a, b], \mathbb{R}\right)$ is defined by

$$
{ }_{a} \mathcal{J}_{t}^{\alpha} \phi(t)=\frac{1}{\Gamma(\alpha)} \int_{a}^{t}(t-s)^{\alpha-1} \phi(s) d s,
$$

provided the integral on right hand side exists.

Definition 2. For a given function $\phi(t) \in C^{n}[a, b]$, the Caputo fractional order derivative of order $\alpha$ is defined as

$$
\begin{aligned}
& D^{\alpha} \phi(t)=\frac{1}{\Gamma(n-\alpha)} \int_{a}^{t} \frac{\phi^{(n)}(s)}{(t-s)^{\alpha+1-n}} d s, \\
& \quad n-1 \leq \alpha<n, n \in N,
\end{aligned}
$$

provided the right side is pointwise defined on $(a, \infty)$, where $n=[\alpha]+1$ in case $\alpha$ is not an integer and $n=\alpha$ in case $\alpha$ is an integer.

Hence, it follows that $I^{\alpha} t^{k}=(\Gamma(1+k) / \Gamma(1+k+$ $\alpha)) t^{k+\alpha}$ for $\alpha>0, k \geq 0, D^{\alpha} C=0$, for a constant $C$, and

$$
D^{\alpha} t^{k}=\frac{\Gamma(1+k)}{\Gamma(1+k-\alpha)} t^{k-\alpha}, \quad \text { for } k \geq[\alpha] .
$$


2.1. The Shifted Jacobi Polynomials. The well known two parametric Jacobi polynomials defined on $[0, \tau]$, with parameters $\alpha$ and $\beta$, are given by the following relation:

$$
P_{\eta, i}^{(\alpha, \beta)}(t)=\sum_{k=0}^{i} \mho_{(i, k)}^{(\alpha, \beta)} t^{k}, \quad i=0,1,2,3, \ldots
$$

where

$$
\mho_{(i, k)}^{(\alpha, \beta)}=\frac{(-1)^{i-k} \Gamma(i+\beta+1) \Gamma(i+k+\alpha+\beta+1)}{\Gamma(k+\beta+1) \Gamma(i+\alpha+\beta+1)(i-k) ! k ! \tau^{k}}
$$

The Jacobi polynomials are orthogonal and the orthogonality condition is

$$
\int_{0}^{\tau} P_{\tau, i}^{(\alpha, \beta)}(t) P_{\tau, j}^{(\alpha, \beta)}(t) w_{\tau}^{(\alpha, \beta)}(t) d t=R_{\tau, j}^{(\alpha, \beta)} \delta_{i, j}
$$

where $w_{\tau}^{(\alpha, \beta)}(t)=(\tau-t)^{\alpha} t^{\beta}$ is the weight function and $R_{\tau, j}^{(\alpha, \beta)}=$ $\tau^{\alpha+\beta+1} \Gamma(j+\alpha+1) \Gamma(j+\beta+1) /(2 j+\alpha+\beta+1) \Gamma(j+1) \Gamma(j+\alpha+\beta+1)$ is known as normalization constant.

Any squared integrable function $v(t)$ on $[0, \tau]$ can be approximated by shifted Jacobi polynomials as follows: $v(t)=$ $\sum_{j=0}^{\infty} c_{j} P_{\eta, j}^{(\alpha, \beta)}(t)$, where the coefficient $c_{j}$ can easily be calculated using (10). In practice we are interested in the truncated series, which can also be written in vector form as

$$
v(t)=\mathbf{H}_{M}^{T} \Psi_{M}(t)
$$

where $M=m+1, \mathbf{H}_{M}^{T}$ is the coefficient vector, and $\Psi_{M}(t)$ is $M$ terms function vector.

One important property of shifted Jacobi polynomials which will be frequently used in our analysis is given as

$$
\max _{x \in[0, \tau]}\left|P_{\tau, i}^{(\alpha, \beta)}(x)\right| \leq \widehat{C}(i, \epsilon)
$$

where $\widehat{C}(i, \epsilon)=\Gamma(i+\epsilon+1) / \Gamma(i+1) \Gamma(\epsilon+1)$ and $\epsilon=\max (\alpha, \beta)$.

Lemma 3. The definite integral of the product of weight function with three Jacobi polynomials over the domain $[0, \tau]$ is constant, defined as $F_{(l, m, n)}^{(i, j, k)}$, defined by

$$
\int_{0}^{\tau} w_{\tau}^{(\alpha, \beta)}(t) P_{\tau, i}^{(\alpha, \beta)}(t) P_{\tau, j}^{(\alpha, \beta)}(t) P_{\tau, k}^{(\alpha, \beta)}(t) d t=F_{(l, m, n)}^{(i, j, k)},
$$

where

$$
\begin{aligned}
& F_{(l, m, n)}^{(i, j, k)}=\sum_{l=0}^{i} \sum_{m=0}^{j} \sum_{n=0}^{k} \mho_{(i, l)}^{(\alpha, \beta)} \mho_{(j, m)}^{(\alpha, \beta)} \mho_{(k, n)}^{(\alpha, \beta)} \Upsilon_{(l, m, n)}, \\
& \Upsilon_{(l, m, n)} \\
& =\frac{\Gamma(l+m+n+\beta+1) \Gamma(\alpha+1) \tau^{(l+m+n+\alpha+\beta+1)}}{\Gamma(l+m+n+\alpha+\beta+1)} .
\end{aligned}
$$

Proof. In view of (8), we obtain

$$
\begin{aligned}
& \int_{0}^{\tau} w_{\tau}^{(\alpha, \beta)}(t) P_{\tau, i}^{(\alpha, \beta)}(t) P_{\tau, j}^{(\alpha, \beta)}(t) P_{\tau, k}^{(\alpha, \beta)}(t) d t \\
& =\sum_{l=0}^{i} \mho_{(i, l)}^{(\alpha, \beta)} \sum_{m=0}^{j} \mho_{(j, m)}^{(\alpha, \beta)} \sum_{n=0}^{k} \mho_{(k, n)}^{(\alpha, \beta)} \int_{0}^{\tau} t^{(l+m+n+\beta)}(\tau-t)^{\alpha} d t .
\end{aligned}
$$

Using convolution theorem of Laplace transform, we have

$$
\begin{aligned}
& \int_{0}^{\tau} t^{(l+m+n+\beta)}(\tau-t)^{\alpha} d t \\
& \quad=\frac{\Gamma(l+m+n+\beta+1) \Gamma(\alpha+1) \tau^{(l+m+n+\alpha+\beta+1)}}{\Gamma(l+m+n+\alpha+\beta+1)} \\
& \quad=\Upsilon_{(l, m, n)} .
\end{aligned}
$$

Using the value of $\Upsilon_{(l, m, n)}$ in (15), we obtain

$$
\int_{0}^{\tau} w_{\tau}^{(\alpha, \beta)}(t) P_{\tau, i}^{(\alpha, \beta)}(t) P_{\tau, j}^{(\alpha, \beta)}(t) P_{\tau, k}^{(\alpha, \beta)}(t) d t=F_{(l, m, n)}^{(i, j, k)}
$$

\section{Operational Matrices}

In this section we first recall some previous results and then derive some new operational matrices. The following results are known [22].

Lemma 4. Let $\Psi_{M}(t)$ be the function vector as defined in (11); then the $\gamma$-order integration of $\Psi_{M}(t)$ is given by $I^{\gamma} \Psi_{M}(t)=$ $\mathbf{H}_{M \times M}^{\tau, \gamma} \boldsymbol{\Psi}_{M}(t)$, where $\mathbf{H}_{M \times M}^{\tau, \gamma}$ is the operational matrix of integration of order $\gamma$. The entries $\Theta_{i, j, k}$ of $\mathbf{H}_{M \times M}^{\tau, \gamma}$ are defined as

$$
\Theta_{i, j, k}=\sum_{k=0}^{i} \Lambda_{i, k, \gamma} S_{j}
$$

where

$$
\begin{aligned}
\Lambda_{i, k, \gamma} & =\frac{(-1)^{i-k} \Gamma(i+\beta+1) \Gamma(i+k+\alpha+\beta+1) \Gamma(1+k)}{\Gamma(k+\beta+1) \Gamma(i+\alpha+\beta+1)(i-k) ! k ! \Gamma(1+k+\gamma) \tau^{k}}, \\
S_{j} & =\sum_{l=0}^{j} \frac{(-1)^{j-l}(2 j+\alpha+\beta+1) \Gamma(j+1) \Gamma(j+l+\alpha+\beta+1) \Gamma(k+\gamma+l+\beta+1) \Gamma(\alpha+1) \tau^{\gamma}}{\Gamma(j+\alpha+1) \Gamma(l+\beta+1)(j-l) ! l ! \Gamma(k+\gamma+l+\beta+\alpha+2)} .
\end{aligned}
$$


Proof. The detailed proof of this lemma is available in [22].

defined as

Lemma 5. Let $\Psi_{M}(t)$ be the function vector as defined in (11); then the $\gamma$-order derivative of $\Psi_{M}(t)$ is given by $D^{\gamma} \Psi_{M}(t)=$ $\mathbf{G}_{M \times M}^{\tau, \gamma} \Psi_{M}(t)$, where $\mathbf{G}_{M \times M}^{\tau, \gamma}$ is the operational matrix of fractional differentiation of order $\gamma$. The entries $\Phi_{i, j, k}$ of $\mathbf{G}_{M \times M}^{\tau, \gamma}$ are

$$
\Phi_{i, j, k}=\sum_{k=\lceil\gamma\rceil}^{i} \Lambda_{i, k, \gamma} S_{j}
$$

where

$$
\begin{aligned}
\Lambda_{i, k, \gamma} & =\frac{(-1)^{i-k} \Gamma(i+\beta+1) \Gamma(i+k+\alpha+\beta+1) \Gamma(1+k)}{\Gamma(k+\beta+1) \Gamma(i+\alpha+\beta+1)(i-k) ! k ! \Gamma(1+k-\gamma) \tau^{k}}, \\
S_{j} & =\sum_{l=0}^{j} \frac{(-1)^{j-l}(2 j+\alpha+\beta+1) \Gamma(j+1) \Gamma(j+l+\alpha+\beta+1) \Gamma(k+\gamma+l+\beta+1) \Gamma(\alpha+1) \tau^{\gamma}}{\Gamma(j+\alpha+1) \Gamma(l+\beta+1)(j-l) ! l ! \Gamma(k+\gamma+l+\beta+\alpha-2)} .
\end{aligned}
$$

Proof. The detailed proof of this lemma is available in [22].

In [22], these operational matrices are used for solutions to coupled systems of initial value problems for fractional order differential equations. These matrices do not have the ability to handle $m$-point nonlocal boundary value problems. Hence the need to develop new operational matrices which have the ability to handle boundary conditions as well as initial conditions has been of great importance. The new operational matrices include the operational matrices studied in [22] as a factor and have ability to solve fractional order boundary value problems.

Lemma 6. Let $u(t)$ and $\phi_{n}(t)$ be any functions defined on $[0, \tau]$. Then

$$
\phi_{n}(t) D^{\sigma} u(t)=\mathbf{W}_{M}^{T} K_{\phi_{n}}^{\sigma} \Psi_{M}(t),
$$

where $\mathbf{W}_{M}^{T}$ is the Jacobi coefficients vector of $u(t)$ defined by (11) and

$$
K_{\phi_{n}}^{\sigma}=\mathbf{G}_{M \times M}^{\tau, \sigma} \mathbf{J}_{M \times M}^{\tau, \phi_{n}}
$$

The matrix $\mathbf{G}_{M \times M}^{\tau, \sigma}$ is the operational matrix of derivative as defined in Lemma 5 and

$$
\mathbf{J}_{M \times M}^{\eta, \phi_{n}}=\left[\Theta_{r+1, s+1}\right], \quad r=0,1, \ldots, m, s=0,1, \ldots, m .
$$

The entries are defined by the relation

$$
\Theta_{r, s}=\frac{1}{R_{\tau, s}^{(\alpha, \beta)}} \sum_{i=0}^{m} c_{i} F_{(l, m, n)}^{(i, r, s)},
$$

where $c_{i}$ are the Jacobi coefficients of $\phi_{n}(t)$ and $F_{(l, m, n)}^{(i, r, s)}$ is as defined in Lemma 3.

Proof. Using (11) and Lemma 5, we obtain

$$
\phi_{n}(t) D^{\sigma} u(t)=\phi_{n}(t) W_{M}^{T} G_{M \times M}^{\tau, \sigma} \Psi_{M}(t),
$$

where $u(t)=W_{M}^{T} \Psi_{M}(t)$. With rearranging, the above equation can be written as

$$
\phi_{n}(t) D^{\sigma} Y(t)=W_{M}^{T} G_{M \times M}^{\tau, \sigma} \overbrace{\Psi_{M}(t)},
$$

where

$$
\overbrace{\Psi_{M}(t)}=\left[\begin{array}{llllllll}
\phi_{n}(t) P_{\tau, 0}^{(\alpha, \beta)}(t) & \phi_{n}(t) P_{\tau, 1}^{(\alpha, \beta)}(t) & \cdots & \phi_{n}(t) P_{\tau, r}^{(\alpha, \beta)}(t) & \cdots & \phi_{n}(t) P_{\tau, m}^{(\alpha, \beta)}(t)
\end{array}\right]^{T} .
$$

Approximating $\phi_{n}(t)$ with Jacobi polynomials, we have

$$
\phi_{n}(t)=\sum_{i=0}^{m} C_{i} P_{\tau, i}^{(\alpha, \beta)}(t) .
$$

Hence (28) takes the form

$$
\overbrace{\Psi_{M}(t)}=\left[\begin{array}{llllll}
\aleph_{0}(t) & \aleph_{1}(t) & \cdots & \aleph_{r}(t) & \cdots & \aleph_{m}(t)
\end{array}\right]^{T},
$$

where

$$
\aleph_{r}(t)=\sum_{i=0}^{m} C_{i} P_{\tau, i}^{(\alpha, \beta)}(t) P_{\tau, r}^{(\alpha, \beta)}(t), \quad r=0,1, \ldots, m .
$$

Now approximating $\aleph_{r}(t)$ with Jacobi polynomials, we have

$$
\aleph_{r}(t)=\sum_{s=0}^{m} d_{s}^{r} P_{\tau, s}^{(\alpha, \beta)}(t),
$$

where

$$
d_{s}^{r}=\frac{1}{R_{\tau, s}^{(\alpha, \beta)}} \int_{0}^{\tau} \aleph_{r}(t) W_{\tau}^{(\alpha, \beta)}(t) P_{\tau, s}^{(\alpha, \beta)}(t) d t .
$$

Using (31) in (33) we obtain

$$
d_{s}^{r}=\frac{1}{R_{\tau, s}^{(\alpha, \beta)}} \sum_{i=0}^{m} c_{i}
$$




$$
\cdot \int_{0}^{\tau} W_{\tau}^{(\alpha, \beta)}(t) P_{\tau, i}^{(\alpha, \beta)}(t) P_{\tau, r}^{(\alpha, \beta)}(t) P_{\tau, s}^{(\alpha, \beta)}(t) d t
$$

which in view of Lemma 3 takes the form

$$
d_{s}^{r}=\frac{1}{R_{\tau, s}^{(\alpha, \beta)}} \sum_{i=0}^{m} c_{i} F_{(l, m, n)}^{(i, r, s)}=\Theta_{r, s}
$$

Repeating the procedure for $r=0,1, \ldots, m$ and $s=$ $0,1, \ldots, m$, we obtain

$$
\overbrace{\Psi_{M}(t)}=\mathbf{J}_{M \times M}^{\eta, \phi_{n}} \Psi_{M}(t) .
$$

Using (36) in (27), we get

$$
\begin{aligned}
\phi_{n}(t) D^{\sigma} u(t) & =\mathbf{W}_{M}^{T} \mathbf{G}_{M \times M}^{\tau, \sigma} \mathbf{J}_{M \times M}^{\tau, \phi_{n}} \Psi_{M}(t) \\
& =\mathbf{W}_{M}^{T} \mathbf{K}_{\phi_{n}}^{\sigma} \Psi_{M}(t) .
\end{aligned}
$$

Lemma 7. For $\phi_{n}^{c}=c t^{n}$, where $c$ and $n$ are real constants, $u(t)=\mathbf{W}_{M}^{T} \Psi_{M}(t)$, and, for $0<\eta \leq \tau$, the following holds:

$$
\phi_{n 00}^{c} I_{\eta}^{\sigma} u(t)=\mathbf{W}_{M}^{T} \mathbf{Q}_{M \times M}^{\left(\sigma, \phi_{n}^{c}, \eta\right)} \Psi_{M}(t),
$$

where $\mathbf{Q}_{M \times M}^{\left(\sigma, \phi_{n}^{c}, \eta\right)}$ is $M \times M$ operational matrix and is defined by

$$
\begin{aligned}
\mathbf{Q}^{\left(\sigma, \phi_{n}^{c}, \eta\right)}=\left[d_{i+1, j+1}\right], & \\
& i=0,1, \ldots, m, j=0,1, \ldots, m .
\end{aligned}
$$

And the entries $d_{(i, j)}$ are defined by the relation

$$
\begin{aligned}
d_{(i, j)}= & \sum_{k=0}^{i} \mho_{(i, k)}^{(\alpha, \beta)} \sum_{l=0}^{j} \mho_{(j, l)}^{(\alpha, \beta)} \\
& \cdot \frac{c \Gamma(1+k) \Gamma(l+\beta+n+1) \Gamma(\alpha+1)}{R_{\eta, j}^{(\alpha, \beta)} \Gamma(1+k+\sigma) \Gamma(l+\beta+n+\alpha+1)} \\
& \cdot \tau^{(l+\beta+n+\alpha+1)}\left(\eta^{k+\sigma}\right) .
\end{aligned}
$$

Proof. Using the definition of fractional integral (5), on

$$
u(t)=\sum_{i=0}^{m} c_{i} P_{\tau, i}^{(\alpha, \beta)}(t)
$$

we obtain

$$
{ }_{0} I_{\eta}^{\sigma} u(t)=\sum_{i=0}^{m} c_{i 0} I_{\eta}^{\sigma} P_{\tau, i}^{(\alpha, \beta)}(t)
$$

which in view of the definition of Jacobi polynomials yields

$$
{ }_{0} I_{\eta}^{\sigma} u(t)=\sum_{i=0}^{m} c_{i} \sum_{k=0}^{i} \mho_{(i, k)}^{(\alpha, \beta)}{ }_{0} I_{\eta}^{\sigma} t^{k}
$$

Evaluating the integral and after simplification, we obtain

$$
{ }_{0} I_{\eta}^{\sigma} u(t)=\sum_{i=0}^{m} c_{i}\left(\sum_{k=0}^{i} \mho_{(i, k)}^{(\alpha, \beta)} \frac{\Gamma(1+k)\left(\eta^{k+\sigma}\right)}{\Gamma(1+k+\sigma)}\right)
$$

For simplicity, use the following notation:

$$
\overbrace{\mho_{(i, k)}^{(\alpha, \beta, \eta, \sigma)}}=\sum_{k=0}^{i} \mho_{(i, k)}^{(\alpha, \beta)} \frac{\Gamma(1+k)\left(\eta^{k+\sigma}\right)}{\Gamma(1+k+\sigma)} .
$$

From (44) and (45), it follows that

$$
\phi_{n 0}^{c} I_{\eta}^{\sigma} u(t)=\sum_{i=0}^{m} c_{i} \overbrace{\mho_{(i, k)}^{(\alpha, \beta, \eta, \sigma)}} c t^{n} .
$$

We may also write

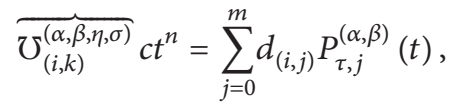

where $d_{(i, j)}$ are given by

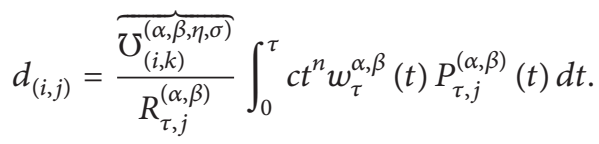

After simplification, we have

$$
\begin{aligned}
& d_{(i, j)}=\frac{c \overbrace{\mho_{(i, k)}^{(\alpha, \beta, \eta, \sigma)}}^{j}}{R_{\tau, j}^{(\alpha, \beta)}} \sum_{l=0}^{j} \mho_{(j, l)}^{(\alpha, \beta)} \int_{0}^{\tau} t^{(l+\beta+n)}(\tau-t)^{\alpha} d t,
\end{aligned}
$$

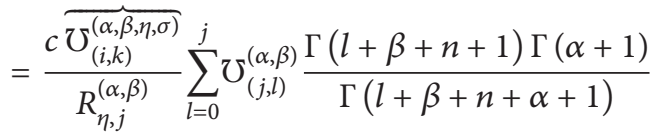

$$
\begin{aligned}
& \text { - } \tau^{(l+\beta+n+\alpha+1)} \text {. }
\end{aligned}
$$

Now using relation (45) we can write

$$
\begin{aligned}
d_{(i, j)}= & \sum_{k=0}^{i} \mho_{(i, k)}^{(\alpha, \beta)} \sum_{l=0}^{j} \mho_{(j, l)}^{(\alpha, \beta)} \\
& \cdot \frac{c \Gamma(1+k) \Gamma(l+\beta+n+1) \Gamma(\alpha+1)}{R_{\tau, j}^{(\alpha, \beta)} \Gamma(1+k+\sigma) \Gamma(l+\beta+n+\alpha+1)} \\
& \cdot \tau^{(l+\beta+n+\alpha+1)}\left(\eta^{k+\sigma}\right) .
\end{aligned}
$$

Using (50) in (46), we obtain

$$
\phi_{n 0}^{c} I_{\eta}^{\sigma} Y(t)=\sum_{i=0}^{m} \sum_{j=0}^{m} C_{i} d_{(i, j)} P_{\tau, j}^{(\alpha, \beta)}(t),
$$

which can be written in matrix form as

$$
\phi_{n 0}^{c} I_{\eta}^{\sigma} Y(t)=K_{M}^{T} Q_{M \times M}^{\left(\sigma, \phi_{n}^{c}, \eta\right)} \Psi_{M}(t),
$$

where the entries of the matrix $Q_{M \times M}^{\left(\sigma, \phi_{n}^{c}, \eta\right)}$ are as defined in (50). 


\section{Application of the Operational Matrices}

In this section we apply the new operational matrices to solve coupled system of nonlinear fractional differential equations. The following lemmas are of basic importance in our further investigation.

Lemma 8. If $D^{\sigma} u(t)=\mathbf{W}_{M}^{T} \Psi_{M}(t)$, where $1<\sigma \leq 2$, and

$$
\begin{aligned}
& u(0)=u_{0}, \\
& u(\tau)=\sum_{i=1}^{m-2} \zeta_{i} u\left(\xi_{i}\right),
\end{aligned}
$$

where $\zeta_{i}$ and $\xi_{i}$ are as defined in (2), and if $\sum_{i=0}^{m-2} \zeta_{i} \xi_{i}-\tau \neq 0$, then $u(t)$ can be written as

$$
u(t)=\mathbf{W}_{M}^{T} \mathbf{V}_{\mathbf{1}} \Psi_{M}(t)
$$

and the matrix $\mathbf{V}_{\mathbf{1}}$ is defined as

$$
\mathbf{V}_{\mathbf{1}}=\left(\mathbf{H}_{M \times M}^{\tau, \sigma}+\mathbf{Q}_{M \times M}^{\left(\sigma, \phi_{1}^{\rho}, \tau\right)}-\sum_{i=1}^{m-2} \mathbf{Q}_{M \times M}^{\left(\sigma, \phi_{1}^{\rho_{i}}, \xi_{i}\right)}+\mathbf{F}_{\mathbf{1} M}^{T}\right),
$$

where $\rho=\tau /\left(\sum_{i=0}^{m-2} \zeta_{i} \xi_{i}-\tau\right), \varrho_{i}=\xi_{i} /\left(\sum_{i=0}^{m-2} \zeta_{i} \xi_{i}-\tau\right)$, and $\mathbf{F}_{\mathbf{1} M}^{T} \boldsymbol{\Psi}_{M}(t)=u_{0}+\left(t /\left(\sum_{i=0}^{m-2} \zeta_{i} \xi_{i}-\tau\right)\right)\left(-\sum_{i=1}^{m-2} \zeta_{i} u_{0}+u_{0}\right)$.

Proof. Consider

$$
D^{\sigma} u(t)=\mathbf{W}_{M}^{T} \Psi_{M}(t) .
$$

Application of fractional integration of order $\sigma$ implies that

$$
u(t)=\mathbf{W}_{M}^{T} \mathbf{H}_{M \times M}^{\tau, \sigma} \Psi_{M}(t)+c_{0}+c_{1} t,
$$

where $c_{0}$ and $c_{1}$ are constants of integration. Using the initial condition $u(0)=u_{0}$ implies that $c_{0}=u_{0}$. Application of nonlocal boundary condition implies that

$$
\begin{aligned}
\sum_{i=1}^{m-2} \zeta_{i} u\left(\xi_{i}\right)= & \mathbf{W}_{M}^{T} \sum_{i=1}^{m-2} \zeta_{i} \mathbf{H}_{M \times M}^{\tau, \sigma} \boldsymbol{\Psi}_{M}\left(\xi_{i}\right)+\sum_{i=1}^{m-2} \zeta_{i} u_{0} \\
& +c_{1} \sum_{i=1}^{m-2} \zeta_{i} \xi_{i}, \\
u(\tau)= & \mathbf{W}_{M}^{T} \mathbf{H}_{M \times M}^{\tau, \sigma} \boldsymbol{\Psi}_{M}(\tau)+u_{0}+c_{1} \tau .
\end{aligned}
$$

Equating the above two equations we get

$$
\begin{aligned}
c_{1} & =\frac{1}{\sum_{i=0}^{m-2} \zeta_{i} \xi_{i}-\tau}\left(\mathbf{W}_{M}^{T} \mathbf{H}_{M \times M}^{\tau, \sigma} \boldsymbol{\Psi}_{M}(\tau)\right. \\
& \left.-\mathbf{W}_{M}^{T} \sum_{i=1}^{m-2} \zeta_{i} \mathbf{H}_{M \times M}^{\tau, \sigma} \boldsymbol{\Psi}_{M}\left(\xi_{i}\right)-\sum_{i=1}^{m-2} \zeta_{i} u_{0}+u_{0}\right) .
\end{aligned}
$$

Using the value of $c_{1}$ in (57) and making use of Lemma 6 we get the following estimates:

$$
\begin{aligned}
u(t)= & \mathbf{W}_{M}^{T} \mathbf{H}_{M \times M}^{\tau, \sigma} \boldsymbol{\Psi}_{M}(t)+\mathbf{W}_{M}^{T} \mathbf{Q}_{M \times M}^{\left(\sigma, \phi_{1}^{\rho}, \tau\right)} \Psi_{M}(t) \\
& -\mathbf{W}_{M}^{T} \sum_{i=1}^{m-2} \mathbf{Q}_{M \times M}^{\left(\sigma, \phi_{1}^{g_{i}}, \xi_{i}\right)} \Psi_{M}(t)+\mathbf{F}_{\mathbf{1} M}^{T} \Psi_{M}(t),
\end{aligned}
$$

where $\rho=1 /\left(\sum_{i=0}^{m-2} \zeta_{i} \xi_{i}-\tau\right), \varrho_{i}=\xi_{i} /\left(\sum_{i=0}^{m-2} \zeta_{i} \xi_{i}-\tau\right)$, and

$$
\mathbf{F}_{\mathbf{1} M}^{T} \boldsymbol{\Psi}_{M}(t)=u_{0}+\frac{t}{\sum_{i=0}^{m-2} \zeta_{i} \xi_{i}-\tau}\left(-\sum_{i=1}^{m-2} \zeta_{i} u_{0}+u_{0}\right) .
$$

After simplification we get

$u(t)$

$$
\begin{aligned}
& =\mathbf{W}_{M}^{T}\left(\mathbf{H}_{M \times M}^{\tau, \sigma}+\mathbf{Q}_{M \times M}^{\left(\sigma, \phi_{1}^{\rho}, \tau\right)}-\sum_{i=1}^{m-2} \mathbf{Q}_{M \times M}^{\left(\sigma, \phi_{1}^{\rho_{i}}, \xi_{i}\right)}+\mathbf{F}_{\mathbf{1} M}^{T}\right) \\
& \cdot \boldsymbol{\Psi}_{M}(t),
\end{aligned}
$$

which completes the proof.

Lemma 9. If $D^{\sigma} u(t)=\mathbf{W}_{M}^{T} \boldsymbol{\Psi}_{M}(t)$, where $1<\sigma \leq 2$ and

$$
\begin{aligned}
& u^{\prime}(0)=a_{1} u(0)+a_{2} u(\tau), \\
& u^{\prime}(\tau)=b_{1} u(0)+b_{2} u(\tau),
\end{aligned}
$$

then

$$
u(t)=\mathbf{W}_{M}^{T} \mathbf{V}_{2} \Psi_{M}(t)
$$

where the matrix $\mathbf{V}_{2}$ is defined as

$$
\begin{aligned}
\mathbf{V}_{\mathbf{2}} & =\left(\mathbf{H}_{M \times M}^{\tau, \sigma}+\mathbf{Q}_{M \times M}^{\left(\sigma, \phi_{0}^{d_{1}}, \tau\right)}+\mathbf{Q}_{M \times M}^{\left(\sigma-1, \phi_{0}^{d_{2}}, \tau\right)}+\mathbf{Q}_{M \times M}^{\left(\sigma, \phi_{1}^{d_{3}}, \tau\right)}\right. \\
& \left.+\mathbf{Q}_{M \times M}^{\left(\sigma-1, \phi_{1}^{d_{4}}, \tau\right)}\right)
\end{aligned}
$$

where $d_{1}=\left(\lambda_{1} \lambda_{3}\left(1-a_{2} \tau\right)-a_{2} \lambda_{2}\right) / \lambda_{2} \lambda_{3}, d_{2}=-\lambda_{3}\left(1-a_{2} \tau\right) / \lambda_{2}$, $d_{3}=\lambda_{1} / \lambda_{2}$, and $d_{4}=-\lambda_{3} / \lambda_{2}$. Here one assumes that $\lambda_{2}, \lambda_{3} \neq$ 0 and are defined as $\lambda_{1}=\left(b_{1}+b_{2}\right) a_{2}-\left(a_{1}+a_{2}\right) b_{2}, \lambda_{2}=\left(b_{1}+\right.$ $\left.b_{2}\right)\left(1-a_{2} \tau\right)-\left(a_{1}+a_{2}\right)\left(1-b_{2} \tau\right)$, and $\lambda_{3}=\left(a_{1}+a_{2}\right)$.

Proof. Consider

$$
D^{\sigma} u(t)=\mathbf{W}_{M}^{T} \boldsymbol{\Psi}_{M}(t)
$$

which implies that

$$
u(t)=\mathbf{W}_{M}^{T} \mathbf{H}_{M \times M}^{\tau, \sigma} \mathbf{\Psi}_{M}(t)+c_{0}+c_{1} t
$$

From the above equation we can also write

$$
u^{\prime}(t)=\mathbf{W}_{M}^{T} \mathbf{H}_{M \times M}^{\tau, \sigma-1} \Psi_{M}(t)+c_{1} .
$$

Now using $u^{\prime}(0)=a_{1} u(0)+a_{2} u(\tau)$ and $u^{\prime}(\tau)=b_{1} u(0)+b_{2} u(\tau)$ we get

$$
\begin{aligned}
& \left(1-a_{2} \tau\right) c_{1}-\left(a_{1}+a_{2}\right) c_{0}=a_{2} \mathbf{W}_{M}^{T} \mathbf{H}_{M \times M}^{\tau, \sigma} \Psi_{M}(\tau) . \\
& \left(1-b_{2} \tau\right) c_{1}-\left(b_{1}+b_{2}\right) c_{0} \\
& \quad=b_{2} \mathbf{W}_{M}^{T} \mathbf{H}_{M \times M}^{\tau, \sigma} \Psi_{M}(\tau)-\mathbf{W}_{M}^{T} \mathbf{H}_{M \times M}^{\tau, \sigma-1} \Psi_{M}(\tau),
\end{aligned}
$$


which can be easily solved for $c_{0}$ and $c_{1}$, and we can write

$$
\begin{aligned}
c_{1}= & \frac{\lambda_{1}}{\lambda_{2}} \mathbf{W}_{M}^{T} \mathbf{H}_{M \times M}^{\tau, \sigma} \Psi_{M}(\tau)-\frac{\lambda_{3}}{\lambda_{2}} \mathbf{W}_{M}^{T} \mathbf{H}_{M \times M}^{\tau, \sigma-1} \Psi_{M}(\tau), \\
c_{0}= & \frac{\lambda_{1} \lambda_{3}\left(1-a_{2} \tau\right)-a_{2} \lambda_{2}}{\lambda_{2} \lambda_{3}} \mathbf{W}_{M}^{T} \mathbf{H}_{M \times M}^{\tau, \sigma} \Psi_{M}(\tau) \\
& -\frac{\lambda_{3}\left(1-a_{2} \tau\right)}{\lambda_{2}} \mathbf{W}_{M}^{T} \mathbf{H}_{M \times M}^{\tau, \sigma-1} \Psi_{M}(\tau),
\end{aligned}
$$

where $\lambda_{1}=\left(b_{1}+b_{2}\right) a_{2}-\left(a_{1}+a_{2}\right) b_{2}, \lambda_{2}=\left(b_{1}+b_{2}\right)\left(1-a_{2} \tau\right)-$ $\left(a_{1}+a_{2}\right)\left(1-b_{2} \tau\right)$, and $\lambda_{3}=\left(a_{1}+a_{2}\right)$.

Now using the value of $c_{0}$ and $c_{1}$ in (67) we get the following estimates:

$$
\begin{aligned}
u(t)= & \mathbf{W}_{M}^{T} \mathbf{H}_{M \times M}^{\tau, \sigma} \boldsymbol{\Psi}_{M}(t)+d_{1} \mathbf{W}_{M}^{T} \mathbf{H}_{M \times M}^{\tau, \sigma} \boldsymbol{\Psi}_{M}(\tau) \\
& +d_{2} \mathbf{W}_{M}^{T} \mathbf{H}_{M \times M}^{\tau, \sigma-1} \boldsymbol{\Psi}_{M}(\tau) \\
& +d_{3} t \mathbf{W}_{M}^{T} \mathbf{H}_{M \times M}^{\tau, \sigma} \boldsymbol{\Psi}_{M}(\tau) \\
& +d_{4} t \mathbf{W}_{M}^{T} \mathbf{H}_{M \times M}^{\tau, \sigma-1} \boldsymbol{\Psi}_{M}(\tau),
\end{aligned}
$$

where $d_{1}=\left(\lambda_{1} \lambda_{3}\left(1-a_{2} \tau\right)-a_{2} \lambda_{2}\right) / \lambda_{2} \lambda_{3}, d_{2}=-\lambda_{3}\left(1-a_{2} \tau\right) / \lambda_{2}$, $d_{3}=\lambda_{1} / \lambda_{2}$, and $d_{4}=-\lambda_{3} / \lambda_{2}$.

In view of Lemma 6 we can write the above relation as

$$
\begin{aligned}
u(t)= & \mathbf{W}_{M}^{T} \mathbf{H}_{M \times M}^{\tau, \sigma} \Psi_{M}(t)+\mathbf{W}_{M}^{T} \mathbf{Q}_{M \times M}^{\left(\sigma, \phi_{0}^{d_{1}}, \tau\right)} \Psi_{M}(t) \\
& +\mathbf{W}_{M}^{T} \mathbf{Q}_{M \times M}^{\left(\sigma-1, \phi_{0}^{d_{2}}, \tau\right)} \Psi_{M}(t) \\
& +\mathbf{W}_{M}^{T} \mathbf{Q}_{M \times M}^{\left(\sigma, \phi_{1}^{d_{3}}, \tau\right)} \Psi_{M}(t) \\
& +\mathbf{W}_{M}^{T} \mathbf{Q}_{M \times M}^{\left(\sigma-1, \phi_{1}^{d_{4}}, \tau\right)} \boldsymbol{\Psi}_{M}(t),
\end{aligned}
$$

which can be written as

$$
\begin{gathered}
u(t)=\mathbf{W}_{M}^{T}\left(\mathbf{H}_{M \times M}^{\tau, \sigma}+\mathbf{Q}_{M \times M}^{\left(\sigma, \phi_{0}^{d_{1}}, \tau\right)}+\mathbf{Q}_{M \times M}^{\left(\sigma-1, \phi_{0}^{d_{2}}, \tau\right)}\right. \\
\left.+\mathbf{Q}_{M \times M}^{\left(\sigma, \phi_{1}^{d_{3}}, \tau\right)}+\mathbf{Q}_{M \times M}^{\left(\sigma-1, \phi_{1}^{d_{4}}, \tau\right)}\right) \Psi_{M}(t),
\end{gathered}
$$

which completes proof of the lemma.

4.1. Couple System of FDEs with Variable Coefficients. Consider the following coupled system of FDEs:

$$
\begin{aligned}
u^{\left(\sigma_{1}\right)}(t)= & \chi_{11}(t) u(t)+\chi_{12}(t) u_{1}^{\gamma}(t)+\chi_{13}(t) v(t) \\
& +\chi_{14}(t) v_{1}^{\gamma}(t)+f(t) \\
v^{\left(\sigma_{1}\right)}(t)= & \chi_{21}(t) u(t)+\chi_{22}(t) u_{1}^{\gamma}(t)+\chi_{23}(t) v(t) \\
& +\chi_{24}(t) v_{1}^{\gamma}(t)+g(t)
\end{aligned}
$$

subject to one of the above discussed boundary conditions ((2) or (4)). Assume that $u^{\left(\sigma_{1}\right)}(t)=\mathbf{X}_{M}^{T} \boldsymbol{\Psi}_{M}(t)$ and $v^{\left(\sigma_{1}\right)}(t)=$
$\mathbf{Y}_{M}^{T} \Psi_{M}(t)$. Then in view of Lemma 8 or Lemma 9 (depends on the given set of boundary conditions) we can write

$$
\begin{aligned}
& u(t)=\mathbf{X}_{M}^{T} \mathbf{V}_{i}^{u} \Psi_{M}(t), \\
& v(t)=\mathbf{Y}_{M}^{T} \mathbf{V}_{i}^{v} \Psi_{M}(t) .
\end{aligned}
$$

Note that the subscript $i$ is used to distinguish between different types of boundary condition. If we are given nonlocal boundary conditions we will use $i=1$ and if we are given mix derivative boundary condition we will use $i=2$. Superscripts $u$ and $v$ are used to distinguish between conditions for $u(t)$ and $v(t)$, respectively. Now in view of Lemma 6 , we can write (74) as

$$
\begin{aligned}
\mathbf{X}_{M}^{T} \boldsymbol{\Psi}_{M}(t)= & \mathbf{X}_{M}^{T} \mathbf{V}_{i}^{u} \mathbf{K}_{\chi_{11}}^{0} \Psi_{M}(t)+\mathbf{X}_{M}^{T} \mathbf{V}_{i}^{u} \mathbf{K}_{\chi_{12}}^{\gamma_{1}} \Psi_{M}(t) \\
& +\mathbf{Y}_{M}^{T} \mathbf{V}_{i}^{v} \mathbf{K}_{\chi_{13}}^{0} \Psi_{M}(t) \\
& +\mathbf{Y}_{M}^{T} \mathbf{V}_{i}^{v} \mathbf{K}_{\chi_{14}}^{\gamma_{1}} \Psi_{M}(t)+\mathbf{F}_{M}^{T} \Psi_{M}(t), \\
\mathbf{Y}_{M}^{T} \boldsymbol{\Psi}_{M}(t)= & \mathbf{X}_{M}^{T} \mathbf{V}_{i}^{u} \mathbf{K}_{\chi_{21}}^{0} \Psi_{M}(t)+\mathbf{X}_{M}^{T} \mathbf{V}_{i}^{u} \mathbf{K}_{\chi_{22}}^{\gamma_{1}} \Psi_{M}(t) \\
& +\mathbf{Y}_{M}^{T} \mathbf{V}_{i}^{v} \mathbf{K}_{\chi_{23}}^{0} \Psi_{M}(t) \\
& +\mathbf{Y}_{M}^{T} \mathbf{V}_{i}^{v} \mathbf{K}_{\chi_{24}}^{\gamma_{1}} \Psi_{M}(t)+\mathbf{G}_{M}^{T} \boldsymbol{\Psi}_{M}(t) .
\end{aligned}
$$

which can be converted into easily solvable matrix equation (by following the same step as in [22] from (51) to (69)). The generalized form of the resulting matrix equation is given as

$$
A X+X B=C
$$

where $A, B$, and $C$ are of compatible size. The vector $X$ is the unknown solution to be determined. The solution method of such type of equations is explained in [26] and a detailed algorithm is presented. MatLab command dlyap uses the same algorithm presented in [26] for solution to such type of matrix equations.

4.2. Convergence Analysis. Consider $\Omega_{i, j}^{u}$ and $\Omega_{i, j}^{v}$ as entries of $\mathbf{V}_{i}^{u}$, and $\mathbf{V}_{i}^{v}$, respectively. Also assume that $\Lambda_{i, j}^{\chi_{11}, \gamma_{1}}$ are entries of $\mathbf{K}_{\chi_{24}}^{\gamma_{1}}$. Now we can write

$$
\begin{aligned}
u^{\left(\sigma_{1}\right)}(t) & =\sum_{i=0}^{\infty} u_{i} P_{\tau, i}^{(\alpha, \beta)}(t) \\
& =\mathbf{X}_{M}^{T} \Psi_{M}(t)+\sum_{i=m+1}^{\infty} u_{i} P_{\tau, i}^{(\alpha, \beta)}(t), \\
v^{\left(\sigma_{2}\right)}(t) & =\sum_{i=0}^{\infty} v_{i} P_{\tau, i}^{(\alpha, \beta)}(t) \\
& =\mathbf{Y}_{M}^{T} \Psi_{M}(t)+\sum_{i=m+1}^{\infty} v_{i} P_{\tau, i}^{(\alpha, \beta)}(t) .
\end{aligned}
$$


Using the above estimates and using the same procedure we can write

$$
\begin{aligned}
& \mathbf{X}_{M}^{T} \boldsymbol{\Psi}_{M}(t)-\mathbf{X}_{M}^{T} \mathbf{V}_{i}^{u} \mathbf{K}_{\chi_{11}}^{0} \Psi_{M}(t)-\mathbf{X}_{M}^{T} \mathbf{V}_{i}^{u} \mathbf{K}_{\chi_{12}}^{\gamma_{1}} \Psi_{M}(t) \\
& -\mathbf{Y}_{M}^{T} \mathbf{V}_{i}^{v} \mathbf{K}_{\chi_{13}}^{0} \Psi_{M}(t)-\mathbf{Y}_{M}^{T} \mathbf{V}_{i}^{v} \mathbf{K}_{\chi_{14}}^{\gamma_{1}} \Psi_{M}(t) \\
& -\mathbf{F}_{M}^{T} \Psi_{M}(t)=R_{u}(t), \\
& \mathbf{Y}_{M}^{T} \boldsymbol{\Psi}_{M}(t)-\mathbf{X}_{M}^{T} \mathbf{V}_{i}^{u} \mathbf{K}_{\chi_{21}}^{0} \Psi_{M}(t)-\mathbf{X}_{M}^{T} \mathbf{V}_{i}^{u} \mathbf{K}_{\chi_{22}}^{\gamma_{1}} \Psi_{M}(t) \\
& -\mathbf{Y}_{M}^{T} \mathbf{V}_{i}^{v} \mathbf{K}_{\chi_{23}}^{0} \Psi_{M}(t)-\mathbf{Y}_{M}^{T} \mathbf{V}_{i}^{v} \mathbf{K}_{\chi_{24}}^{\gamma_{1}} \Psi_{M}(t) \\
& -\mathbf{G}_{M}^{T} \boldsymbol{\Psi}_{M}(t)=R_{v}(t),
\end{aligned}
$$

where

$$
\begin{array}{ll}
R_{u}(t)=\sum_{i=m+1}^{\infty} u_{i} \sum_{h=0}^{m} \Omega_{i, h}^{u} \sum_{j=0}^{m} \Lambda_{h, j}^{\chi_{11}, 0} P_{\tau, j}^{(\alpha, \beta)}(t)+\sum_{i=m+1}^{\infty} u_{i} & \cdot \sum_{h=0}^{m} \sum_{j=0}^{m}\left|\Omega_{i, h}^{v} \Lambda_{h, j}^{\chi_{23}, 0} \widehat{C}(j, \epsilon)\right|+\sum_{i=m+1}^{\infty}\left|v_{i}\right| \\
\cdot \sum_{h=0}^{m} \Omega_{i, h}^{u} \sum_{j=0}^{m} \Lambda_{h, j}^{\chi_{12}, \gamma_{1}} P_{\tau, j}^{(\alpha, \beta)}(t) \sum_{i=m+1}^{\infty} v_{i} \sum_{h=0}^{m} \Omega_{i, h}^{v} & \cdot \sum_{h=0}^{m} \sum_{j=0}^{m}\left|\Omega_{i, h}^{v} \Lambda_{h, j}^{\chi_{24}, \gamma_{1}} \widehat{C}(j, \epsilon)\right|+\sum_{i=m+1}^{\infty}\left|g_{i}\right||\widehat{C}(i, \epsilon)| \\
\cdot \sum_{j=0}^{m} \Lambda_{h, j}^{\chi_{13}, 0} P_{\tau, j}^{(\alpha, \beta)}(t)+\sum_{i=m+1}^{\infty} v_{i} \sum_{h=0}^{m} \Omega_{i, h}^{v} & -\sum_{i=m+1}^{\infty}\left|u_{i}\right||\widehat{C}(i, \epsilon)| .
\end{array}
$$$$
\cdot \sum_{j=0}^{m} \Lambda_{h, j}^{\chi_{14}, \gamma_{1}} P_{\tau, j}^{(\alpha, \beta)}(t)+\sum_{i=m+1}^{\infty} f_{i} P_{\tau, i}^{(\alpha, \beta)}(t)
$$$$
-\sum_{i=m+1}^{\infty} u_{i} P_{\tau, i}^{(\alpha, \beta)}(t)
$$$$
R_{v}(t)=\sum_{i=m+1}^{\infty} u_{i} \sum_{h=0}^{m} \Omega_{i, h}^{u} \sum_{j=0}^{m} \Lambda_{h, j}^{\chi_{21}, 0} P_{\tau, j}^{(\alpha, \beta)}(t)+\sum_{i=m+1}^{\infty} u_{i}
$$$$
\cdot \sum_{h=0}^{m} \Omega_{i, h}^{u} \sum_{j=0}^{m} \Lambda_{h, j}^{\chi_{22}, \gamma_{1}} P_{\tau, j}^{(\alpha, \beta)}(t) \sum_{i=m+1}^{\infty} v_{i} \sum_{h=0}^{m} \Omega_{i, h}^{v}
$$$$
\cdot \sum_{j=0}^{m} \Lambda_{h, j}^{\chi_{23}, 0} P_{\tau, j}^{(\alpha, \beta)}(t)+\sum_{i=m+1}^{\infty} v_{i} \sum_{h=0}^{m} \Omega_{i, h}^{v}
$$$$
\cdot \sum_{j=0}^{m} \Lambda_{h, j}^{\chi_{24}, \gamma_{1}} P_{\tau, j}^{(\alpha, \beta)}(t)+\sum_{i=m+1}^{\infty} g_{i} P_{\tau, i}^{(\alpha, \beta)}(t)
$$$$
-\sum_{i=m+1}^{\infty} u_{i} P_{\tau, i}^{(\alpha, \beta)}(t)
$$

where $f_{i}$ and $g_{i}$ are the approximation coefficients of $f(t)$ and $g(t)$, respectively. In view of maximum norm and (12) we can write

$$
\begin{aligned}
& \left\|R_{u}(t)\right\| \leq \sum_{i=m+1}^{\infty}\left|u_{i}\right| \sum_{h=0}^{m} \sum_{j=0}^{m}\left|\Omega_{i, h}^{u} \Lambda_{h, j}^{\chi_{11}, 0} \widehat{C}(j, \epsilon)\right| \\
& +\sum_{i=m+1}^{\infty}\left|u_{i}\right| \sum_{h=0}^{m} \sum_{j=0}^{m}\left|\Omega_{i, h}^{u} \Lambda_{h, j}^{\chi_{12}, \gamma_{1}} \widehat{C}(j, \epsilon)\right| \sum_{i=m+1}^{\infty}\left|v_{i}\right|
\end{aligned}
$$

$$
\begin{aligned}
& \cdot \sum_{h=0}^{m} \sum_{j=0}^{m}\left|\Omega_{i, h}^{v} \Lambda_{h, j}^{\chi_{13}, 0} \widehat{C}(j, \epsilon)\right|+\sum_{i=m+1}^{\infty}\left|v_{i}\right| \\
& \cdot \sum_{h=0}^{m} \sum_{j=0}^{m}\left|\Omega_{i, h}^{v} \Lambda_{h, j}^{\chi_{14}, \gamma_{1}} \widehat{C}(j, \epsilon)\right|+\sum_{i=m+1}^{\infty}\left|f_{i}\right||\widehat{C}(i, \epsilon)| \\
& \quad-\sum_{i=m+1}^{\infty}\left|u_{i}\right||\widehat{C}(i, \epsilon)|, \\
& \left\|R_{v}(t)\right\|=\sum_{i=m+1}^{\infty}\left|u_{i}\right| \sum_{h=0}^{m} \sum_{j=0}^{m}\left|\Omega_{i, h}^{u} \Lambda_{h, j}^{\chi_{21}, 0} \widehat{C}(j, \epsilon)\right| \\
& +\sum_{i=m+1}^{\infty}\left|u_{i}\right| \sum_{h=0}^{m} \sum_{j=0}^{m}\left|\Omega_{i, h}^{u} \Lambda_{h, j}^{\chi_{22}, \gamma_{1}} \widehat{C}(j, \epsilon)\right| \sum_{i=m+1}^{\infty}\left|v_{i}\right|
\end{aligned}
$$

In view of Lemma 2.1 of [22] the expansion coefficients $u_{i}$ and $v_{i}$ decay faster than any of the algebraic order of index $i$. We can write $u_{i}, v_{i}, f_{i}$, and $g_{i}$ approach to zero as $i \rightarrow \infty$ and as a result we conclude that

$$
\begin{gathered}
\left\|R_{u}(t)\right\| \longrightarrow 0 \quad \text { as } m \longrightarrow \infty, \\
\left\|R_{v}(t)\right\| \longrightarrow 0 \quad \text { as } m \longrightarrow \infty .
\end{gathered}
$$

We see that the convergence of the scheme depends on the decay of expansion coefficients, and the decay of expansion coefficients depends solely on the smoothness of solution for the problem. Therefore if the solution for the problem is smooth we get high accuracy at small scale level, and the accuracy will increase if we increase the scale level.

4.3. Nonlinear FDEs with m-Point Boundary Conditions. To solve nonlinear FDEs we will implement operational matrix method combined with qasilinearization method. Consider the following nonlinear FDE:

$$
\begin{aligned}
& u^{\sigma_{1}}=f\left(t, u^{\gamma_{1}}, v^{\gamma_{1}}, u, v\right), \\
& v^{\sigma_{2}}=g\left(t, u^{\gamma_{1}}, v^{\gamma_{1}}, u, v\right) .
\end{aligned}
$$

In qasilinearization method the nonlinear differential equation is converted into linear differential equation with variable coefficients. The nonlinear part of the differential equation is linearized about some function. In most cases the initial function is selected according to the physical problem under consideration, although in some cases it is selected as 
TABLE 1: Comparison of absolute error of Example 1 obtained with the proposed method and its comparison with Haar wavelets [28] and RKM [29].

\begin{tabular}{lccccc}
\hline & $M=8(\mathrm{HW})[28]$ & $n=8(\mathrm{RKM})[29]$ & $M=8(\mathrm{PM})$ & $M=32(\mathrm{HW})[28]$ & $4.42 \times 10^{-7}$ \\
\hline$t=0.1$ & $6.97 \times 10^{-5}$ & $2.41 \times 10^{-5}$ & $4.192 \times 10^{-6}$ & $1.78 \times 10^{-7}$ & $6.05 \times 10^{-9}$ \\
$t=0.2$ & $4.59 \times 10^{-5}$ & $2.10 \times 10^{-5}$ & $2.13 \times 10^{-5}$ & $1.80 \times 10^{-7}$ & $3.15 \times 10^{-9}$ \\
$t=0.3$ & $4.59 \times 10^{-5}$ & $3.42 \times 10^{-6}$ & $1.47 \times 10^{-5}$ & $4.42 \times 10^{-7}$ & $4.04 \times 10^{-9}$ \\
$t=0.4$ & $1.33 \times 10^{-4}$ & $2.23 \times 10^{-5}$ & $4.45 \times 10^{-6}$ & $5.35 \times 10^{-7}$ & $4.04 \times 10^{-9}$ \\
$t=0.5$ & $1.37 \times 10^{-4}$ & $2.26 \times 10^{-5}$ & $2.28 \times 10^{-5}$ & $4.41 \times 10^{-7}$ & $7.10 \times 10^{-9}$ \\
$t=0.6$ & $1.13 \times 10^{-4}$ & $2.93 \times 10^{-5}$ & $9.55 \times 10^{-6}$ & $1.78 \times 10^{-7}$ & $1.98 \times 10^{-9}$ \\
$t=0.7$ & $5.80 \times 10^{-5}$ & $3.27 \times 10^{-5}$ & $1.09 \times 10^{-5}$ & $1.78 \times 10^{-7}$ & $8.82 \times 10^{-9}$ \\
$t=0.8$ & $6.68 \times 10^{-5}$ & $3.00 \times 10^{-5}$ & $1.36 \times 10^{-5}$ & $4.40 \times 10^{-7}$ & $1.34 \times 10^{-8}$ \\
$t=0.9$ & $6.27 \times 10^{-4}$ & $6.15 \times 10^{-5}$ & $1.50 \times 10^{-5}$ & &
\end{tabular}

the solution for the linear part [27]. We will first solve the linear part of (83):

$$
\begin{aligned}
& u^{\sigma_{1}}=L_{1}\left(u, v, u_{1}^{\gamma}, v_{1}^{\gamma}\right), \\
& v^{\sigma_{2}}=L_{2}\left(u, v, u_{1}^{\gamma}, v_{1}^{\gamma}\right),
\end{aligned}
$$

under given boundary conditions using the method developed in the previous section. $L_{1}$ and $L_{2}$ are the linear part of $f$ and $g$, respectively. The solution for this problem will be labeled as $u_{0}(t)$ and $v_{0}(t)$. The next step is to linearize the nonlinear part of $f$ and $g$ about $u_{0}(t)$ and $v_{0}(t)$ using Taylor series expansion. As a result the nonlinear differential equation will be converted into linear differential equation with variable coefficients, which can be solved by the proposed. The whole process can be seen as a recurrence relation as

$$
\begin{aligned}
u_{r+1}^{\sigma_{1}}= & f_{r}+\frac{\partial f_{r}}{\partial u}\left(u_{r+1}-u_{r}\right) \\
& +\frac{\partial f_{r}}{\partial v}\left(v_{r+1}-v_{r}(t)\right) \frac{\partial f_{r}}{\partial u^{\gamma_{1}}}\left(u_{r+1}^{\gamma_{1}}-u_{r}^{\gamma_{1}}\right) \\
& +\frac{\partial f_{r}}{\partial v^{\gamma_{1}}}\left(v_{r+1}^{\gamma_{1}}-v_{r}^{\gamma_{1}}\right), \\
v_{r+1}^{\sigma_{1}}= & g_{r}+\frac{\partial g_{r}}{\partial u}\left(u_{r+1}-u_{r}\right) \\
& +\frac{\partial g_{r}}{\partial v}\left(v_{r+1}-v_{r}(t)\right) \frac{\partial g_{r}}{\partial u^{\gamma_{1}}}\left(u_{r+1}^{\gamma_{1}}-u_{r}^{\gamma_{1}}\right) \\
& +\frac{\partial g_{r}}{\partial v^{\gamma_{1}}}\left(v_{r+1}^{\gamma_{1}}-v_{r}^{\gamma_{1}}\right),
\end{aligned}
$$

where $f_{r}=f\left(t, u_{r}^{\gamma_{1}}, v_{r}^{\gamma_{1}}, u_{r}, v_{r}\right)$ and $g_{r}=g\left(t, u_{r}^{\gamma_{1}}, v_{r}^{\gamma_{1}}, u_{r}, v_{r}\right)$. The above equation is linear fractional differential equations with variable coefficients and can be easily solved with the method developed in the previous section.

\section{Illustrative Examples}

We show the applicability of the method by solving some test problems. Where available we compare our results with results obtained with other methods.
Example 1. Consider the following linear fractional order three-point boundary value problem [28]:

$$
\begin{aligned}
D^{3 / 2} X(t)+\frac{e^{-3 \pi}}{\sqrt{\pi}} X(t) & =g(t), \quad t \in[0,1], \\
X(0) & =0, \\
X(1) & =\frac{-125}{196} X\left(\frac{2}{5}\right),
\end{aligned}
$$

where the forcing term $g(t)$ is defined as

$$
\begin{aligned}
& g(t)=\frac{e^{-3 \pi}}{\sqrt{\pi}}\left(t^{2}\left(40 t^{2}-74 t+33\right)\right. \\
&\left.+4 e^{3 \pi} \sqrt{t}\left(128 t^{2}-148 t+33\right)\right) .
\end{aligned}
$$

The exact solution for this problem is $X(t)=t^{5}-37 t^{3} / 20+$ $33 t^{2} / 40$. This problem is solved in [28] using Haar wavelets (HW). In [29] this problem is solved using improved reproducing kernel method (RKM). We compare the absolute error obtained with the proposed method with the error obtained with HW and RKM. The results are shown in Table 1.

Example 2. Consider the following fractional differential equations having variable coefficients and five-point boundary conditions $[29,30]$ :

$$
\begin{aligned}
D^{1.3} X(t)+\cos (t) D X(t)+2 X(t) & =f(t), \\
X(0) & =0, \\
X\left(\frac{1}{8}\right)+2 X\left(\frac{1}{2}\right)+\frac{31}{49} X\left(\frac{7}{8}\right) & =X(1),
\end{aligned}
$$

where $f(t)=2 t^{2}+2 t \cos (t)+\Gamma(3) / \Gamma(1.7) t^{0.7}$. The exact solution for the problem is $X(t)=t^{2}$. We approximate solution for this problem with the proposed method and compare its absolute error with the error reported in [29, 30] (in these references reproducing kernel method and improved reproducing kernel method are used to solve this problem). We observe that the solution obtained with 
TABLE 2: Comparison of absolute error of Example 2 obtained with the proposed method and its comparison with RKM [30] and improved reproducing kernel method [29].

\begin{tabular}{|c|c|c|c|}
\hline & $M=10(\mathrm{RKM})[30]$ & $M=10$ (IRKM) [29] & $M=20(\mathrm{PM})$ \\
\hline$t=0.1$ & $1.11 \times 10^{-7}$ & $3.09 \times 10^{-8}$ & $7.35 \times 10^{-8}$ \\
\hline$t=0.2$ & $2.87 \times 10^{-7}$ & $1.69 \times 10^{-8}$ & $2.43 \times 10^{-9}$ \\
\hline$t=0.3$ & $5.51 \times 10^{-7}$ & $3.55 \times 10^{-9}$ & $9.54 \times 10^{-9}$ \\
\hline$t=0.4$ & $9.23 \times 10^{-7}$ & $1.89 \times 10^{-9}$ & $2.17 \times 10^{-10}$ \\
\hline$t=0.5$ & $1.41 \times 10^{-6}$ & $2.94 \times 10^{-10}$ & $9.37 \times 10^{-11}$ \\
\hline$t=0.6$ & $2.03 \times 10^{-6}$ & $8.47 \times 10^{-9}$ & $7.58 \times 10^{-11}$ \\
\hline$t=0.7$ & $2.77 \times 10^{-6}$ & $2.97 \times 10^{-8}$ & $4.72 \times 10^{-11}$ \\
\hline$t=0.8$ & $3.64 \times 10^{-6}$ & $3.94 \times 10^{-8}$ & $8.24 \times 10^{-12}$ \\
\hline$t=0.9$ & $4.62 \times 10^{-6}$ & $5.23 \times 10^{-8}$ & $3.57 \times 10^{-10}$ \\
\hline
\end{tabular}

the proposed method is in good agreement with the exact solution. In Table 2 absolute error at different value of $t$ is compared with the error reported in $[29,30]$.

Example 3. Consider the following nonlinear fractional differential equations with 6-point nonlocal boundary value problem:

$$
\begin{aligned}
& D^{1.8} X(t)+X^{\prime}(t)+X(t)=\left(X(t)+X^{\prime}(t)\right)^{2} \\
& \quad-X(t) X^{\prime}(t)+f(t), \quad t \in[0,1], \\
& X(0)=1, \\
& \left(\frac{1}{2}\right) X\left(\frac{1}{2}\right)+\left(\frac{2}{3}\right) X\left(\frac{5}{8}\right)+\left(\frac{1}{4}\right) X\left(\frac{3}{4}\right) \\
& \quad-0.646 X\left(\frac{7}{8}\right)=X(1),
\end{aligned}
$$

where the forcing term $f(t)$ is defined as

$$
\begin{aligned}
f(t)= & 20 t^{4}-4 t^{2}-2 t+11 t^{5}+4 t^{6}-25 t^{8}-5 t^{9}-t^{10} \\
& +\frac{2229536516744740625 t^{16 / 5}}{144115188075855872}-4
\end{aligned}
$$

The exact and unique solution for the problem is $X(t)=$ $t^{5}-2 t+1$. We approximate the solution for this problem with the proposed iterative method. This example is solved using the parameters $\alpha=0, \beta=2$, and $M=8$. We observe that the method provides good approximation to solution for the problem. The approximate solution approaches the exact solution as we make iteration. Absolute difference of exact and approximate solution at different stages of iteration is shown in Table 3. It can be easily seen that at fourth iteration the absolute difference is less than $10^{-8}$.

Example 4. Consider the following nonlinear differential equation with 6-point nonlocal boundary conditions:

$$
\begin{aligned}
& D^{2} X(t)+X^{\prime}(t)+X(t) \\
& \quad=\left(X^{\prime}(t)\right)^{2}+X(t) X^{\prime}(t)+f(t), \quad t \in[0,1],
\end{aligned}
$$

TABLE 3: Absolute difference of exact and approximate solution of Example 3 at different stages of the iteration.

\begin{tabular}{lllll}
\hline & 1st iteration & 2nd iteration & 3rd iteration & 4th iteration \\
\hline$t=0.1$ & $4.42 \times 10^{-2}$ & $1.87 \times 10^{-3}$ & $9.17 \times 10^{-6}$ & $1.65 \times 10^{-8}$ \\
$t=0.2$ & $4.18 \times 10^{-2}$ & $3.04 \times 10^{-3}$ & $1.12 \times 10^{-6}$ & $2.03 \times 10^{-8}$ \\
$t=0.3$ & $2.70 \times 10^{-2}$ & $3.56 \times 10^{-3}$ & $2.30 \times 10^{-6}$ & $1.80 \times 10^{-8}$ \\
$t=0.4$ & $1.42 \times 10^{-2}$ & $3.93 \times 10^{-3}$ & $2.92 \times 10^{-6}$ & $2.77 \times 10^{-8}$ \\
$t=0.5$ & $6.92 \times 10^{-3}$ & $4.09 \times 10^{-3}$ & $3.25 \times 10^{-6}$ & $2.70 \times 10^{-8}$ \\
$t=0.6$ & $3.83 \times 10^{-3}$ & $4.04 \times 10^{-3}$ & $3.33 \times 10^{-6}$ & $2.04 \times 10^{-8}$ \\
$t=0.7$ & $2.81 \times 10^{-3}$ & $3.96 \times 10^{-3}$ & $3.26 \times 10^{-6}$ & $2.52 \times 10^{-8}$ \\
$t=0.8$ & $2.76 \times 10^{-3}$ & $3.80 \times 10^{-3}$ & $3.15 \times 10^{-6}$ & $2.85 \times 10^{-8}$ \\
$t=0.9$ & $3.33 \times 10^{-3}$ & $3.57 \times 10^{-3}$ & $2.95 \times 10^{-6}$ & $1.94 \times 10^{-8}$ \\
$t=1.0$ & $4.58 \times 10^{-3}$ & $3.30 \times 10^{-3}$ & $2.71 \times 10^{-6}$ & $2.48 \times 10^{-8}$ \\
\hline
\end{tabular}

TABLE 4: Absolute difference of exact and approximate solution of Example 4 at different stages of the iteration.

\begin{tabular}{lllll}
\hline & 1st iteration & 2nd iteration & 3rd iteration & 4th iteration \\
\hline$t=0.1$ & $1.61 \times 10^{-5}$ & $5.27 \times 10^{-8}$ & $3.10 \times 10^{-15}$ & $1.24 \times 10^{-17}$ \\
$t=0.2$ & $2.80 \times 10^{-5}$ & $8.29 \times 10^{-8}$ & $4.88 \times 10^{-15}$ & $2.82 \times 10^{-17}$ \\
$t=0.3$ & $3.62 \times 10^{-5}$ & $9.93 \times 10^{-8}$ & $5.63 \times 10^{-15}$ & $2.23 \times 10^{-17}$ \\
$t=0.4$ & $4.13 \times 10^{-5}$ & $1.07 \times 10^{-7}$ & $2.16 \times 10^{-15}$ & $1.26 \times 10^{-16}$ \\
$t=0.5$ & $4.41 \times 10^{-5}$ & $1.11 \times 10^{-7}$ & $5.09 \times 10^{-15}$ & $1.12 \times 10^{-17}$ \\
$t=0.6$ & $4.52 \times 10^{-5}$ & $1.13 \times 10^{-7}$ & $6.21 \times 10^{-15}$ & $2.22 \times 10^{-17}$ \\
$t=0.7$ & $4.53 \times 10^{-5}$ & $1.13 \times 10^{-7}$ & $6.10 \times 10^{-15}$ & $2.22 \times 10^{-17}$ \\
$t=0.8$ & $4.48 \times 10^{-5}$ & $1.12 \times 10^{-7}$ & $5.88 \times 10^{-15}$ & $1.01 \times 10^{-17}$ \\
$t=0.9$ & $4.40 \times 10^{-5}$ & $1.10 \times 10^{-7}$ & $5.77 \times 10^{-15}$ & $4.42 \times 10^{-17}$ \\
$t=1.0$ & $4.28 \times 10^{-5}$ & $1.08 \times 10^{-7}$ & $5.44 \times 10^{-15}$ & $2.21 \times 10^{-17}$ \\
\hline
\end{tabular}

$$
\begin{aligned}
& X(0)=0 \\
& 0.8 X\left(\frac{1}{2}\right)+0.75 X\left(\frac{5}{8}\right)+0.50 X\left(\frac{3}{4}\right)-1.11 X\left(\frac{7}{8}\right) \\
& \quad=X(1) .
\end{aligned}
$$

The forcing term $f(t)$ is defined as

$$
\begin{aligned}
f(t)= & -\frac{\sin (t / 3)^{2}}{9}-\frac{2 \sin (t / 3) \sin (t / 6)^{2}}{3} \\
& -\frac{16 \sin (t / 6)^{2}}{9}+\frac{8}{9} .
\end{aligned}
$$

The exact solution for the problem is $X(t)=\cos (t / 3)$. We carry out the same analysis as in the previous example. The same conclusion is made; the solution converges rapidly to the exact solution for the problem. In Table 4 absolute error at different stages of iteration is given. For this example the absolute error is less than $10^{-16}$ at fourth iteration. For this example we set $\alpha=1, \beta=1$, and $M=10$.

Example 5. Consider the Lane-Emden equations [31]:

$$
\begin{aligned}
& u^{(\sigma)}(t)+\phi_{1}(t) u^{\prime}(t)-c_{11} u^{2}(t)-c_{12} u(t) v(t)=0, \\
& v^{(\sigma)}(t)+\phi_{2}(t) v^{\prime}(t)-c_{21} u^{2}(t)-c_{22} u(t) v(t)=0 .
\end{aligned}
$$


TABLE 5: Maximal error of Example 5 obtained with the proposed method and its comparison with error reported in [31].

\begin{tabular}{lcccc}
\hline & $\mathrm{MER}_{n}^{u}[31]$ & $\mathrm{MER}_{n}^{v}[31]$ & $\mathrm{MER}_{n}^{u}(\mathrm{PM})$ & $\mathrm{MER}_{n}^{v}(\mathrm{PM})$ \\
\hline$n=2$ & 0.86666 & 1.14667 & 1.12626 & 3.92536 \\
$n=3$ & 0.44282 & 0.57063 & 0.59223 & 2.63829 \\
$n=4$ & 0.23094 & 0.29382 & 0.01280 & 0.89223 \\
$n=5$ & 0.12671 & 0.15988 & $1.12 \times 10^{-3}$ & $7.29 \times 10^{-2}$ \\
$n=6$ & 0.07155 & 0.08982 & $9.36 \times 10^{-3}$ & $4.96 \times 10^{-2}$ \\
$n=7$ & 0.04160 & 0.05202 & $3.10 \times 10^{-3}$ & $9.41 \times 10^{-3}$ \\
$n=8$ & 0.02467 & 0.03077 & $8.72 \times 10^{-3}$ & $8.67 \times 10^{-3}$ \\
$n=9$ & 0.01490 & 0.01855 & $6.94 \times 10^{-3}$ & $6.62 \times 10^{-4}$ \\
$n=10$ & 0.00913 & 0.01134 & $1.76 \times 10^{-3}$ & $1.93 \times 10^{-4}$ \\
$n=11$ & 0.00566 & 0.00702 & $6.82 \times 10^{-4}$ & $3.97 \times 10^{-5}$ \\
$n=12$ & 0.00354 & 0.00439 & $7.32 \times 10^{-5}$ & $1.35 \times 10^{-5}$ \\
$n=13$ & 0.00224 & 0.00277 & $2.36 \times 10^{-5}$ & $6.78 \times 10^{-5}$ \\
$n=14$ & 0.00142 & 0.00176 & $7.91 \times 10^{-6}$ & $0.41 \times 10^{-6}$ \\
$n=15$ & 0.00091 & 0.00112 & $9.45 \times 10^{-7}$ & $8.93 \times 10^{-6}$ \\
$n=16$ & 0.00058 & 0.00072 & $7.34 \times 10^{-7}$ & $8.54 \times 10^{-6}$ \\
\hline
\end{tabular}

Systems of Lane-Emden equations arise in the modeling of several physical phenomena, such as pattern formation, population evolution, and chemical reactions. The parameters $c_{11}$, $c_{12}, c_{21}$, and $c_{22}$ can be considered from the actual chemical reaction or dynamics under consideration. We solve this problem with the proposed method subject to the following type of boundary conditions:

$$
\begin{aligned}
& u^{\prime}(0)=2 u(0)-u(1), \\
& u^{\prime}(1)=u(0)+2 u(1), \\
& v^{\prime}(0)=-2 v(0)+v(1), \\
& v^{\prime}(1)=v(0)-2 v(\tau) .
\end{aligned}
$$

In [31], Adomian decomposition method is employed to get approximate solution to this problem. We compare our results with the results reported in [31]. We observe that the current method provides a very good approximation to the solutions for the problem. We calculate the error remainder term:

$$
\begin{aligned}
\operatorname{ER}_{n}^{u}= & u_{n}^{(\sigma)}(t)+\phi_{1}(t) u_{n}^{\prime}(t)-c_{11} u_{n}^{2}(t) \\
& -c_{12} u_{n}(t) v_{n}(t), \\
\operatorname{ER}_{n}^{v}= & v_{n}^{(\sigma)}(t)+\phi_{2}(t) v_{n}^{\prime}(t)-c_{21} u_{n}^{2}(t) \\
& -c_{22} u_{n}(t) v_{n}(t),
\end{aligned}
$$

where $u_{n}$ and $v_{n}$ are the approximate solution for the problem at different stages of iteration $n$. We calculate the maximal error remainder term:

$$
\begin{aligned}
\operatorname{MER}_{n}^{u} & =\max _{t \in[0,1]} \mathrm{ER}_{n}^{u}, \\
\operatorname{MER}_{n}^{v} & =\max _{t \in[0,1]} \mathrm{ER}_{n}^{v} .
\end{aligned}
$$

The comparison of results of the proposed method with Adomain decomposition is presented in Table 5.

\section{Conclusion and Future Work}

Form the above analysis and observation we conclude that the method works very well and efficiently solve fractional order linear and nonlinear differential equation and coupled system under multipoint nonlocal boundary conditions and mixed derivative boundary conditions. When the absolute error is compared with reproducing kernel method and Adomain decomposition method we observe that the method yields very accurate solution. In this work shifted Jacobi polynomials are used; it may also be possible that the reader may get more accurate solution by using another class of orthogonal polynomials like Bernstein polynomials or Hermite polynomials and so forth. Our future work is related to investigating the best choice of orthogonal polynomials.

\section{Competing Interests}

The authors declare that they have no competing interests.

\section{References}

[1] I. Podlubny, Fractional Differential Equations, vol. 198 of Mathematics in Science and Engineering, Academic Press, San Diego, Calif, USA, 1999.

[2] R. Ma, "Multiple positive solutions for nonlinear $m$-point boundary value problems," Applied Mathematics and Computation, vol. 148, no. 1, pp. 249-262, 2004.

[3] B. Ahmad, "Approximation of solutions of the forced Duffing equation with $m$-point boundary conditions," Communications in Applied Analysis, vol. 13, no. 1, pp. 11-20, 2009.

[4] M. Lakestani, M. Dehghan, and S. Irandoust-Pakchin, "The construction of operational matrix of fractional derivatives using B-spline functions," Communications in Nonlinear Science and Numerical Simulation, vol. 17, no. 3, pp. 1149-1162, 2012.

[5] S. A. Yousefi, M. Behroozifar, and M. Dehghan, "Numerical solution of the nonlinear age-structured population models by using the operational matrices of Bernstein polynomials," Applied Mathematical Modelling, vol. 36, no. 3, pp. 945-963, 2012.

[6] M. R. Eslahchi and M. Dehghan, "Application of Taylor series in obtaining the orthogonal operational matrix," Computers \& Mathematics with Applications, vol. 61, no. 9, pp. 2596-2604, 2011.

[7] A. Saadatmandi and M. Dehghan, "A tau approach for solution of the space fractional diffusion equation," Computers \& Mathematics with Applications, vol. 62, no. 3, pp. 1135-1142, 2011.

[8] M. Dehghan and F. Shakeri, "A semi-numerical technique for solving the multi-point boundary value problems and engineering applications," International Journal of Numerical Methods for Heat and Fluid Flow, vol. 21, no. 7, pp. 794-809, 2011.

[9] A. Kayedi-Bardeh, M. R. Eslahchi, and M. Dehghan, "A method for obtaining the operational matrix of fractional Jacobi functions and applications," Journal of Vibration and Control, vol. 20, no. 5, pp. 736-748, 2014.

[10] A. H. Bhrawy and A. S. Alofi, "A Jacobi-Gauss collocation method for solving nonlinear Lane-Emden type equations," Communications in Nonlinear Science and Numerical Simulation, vol. 17, no. 1, pp. 62-70, 2012.

[11] A. H. Bhrawy and M. M. Al-Shomrani, "A shifted Legendre spectral method for fractional-order multi-point boundary 
value problems," Advances in Difference Equations, vol. 2012, no. 1, article 8, pp. 1-19, 2012.

[12] E. H. Doha, A. H. Bhrawy, and S. S. Ezz-Eldien, "A Chebyshev spectral method based on operational matrix for initial and boundary value problems of fractional order," Computers \& Mathematics with Applications, vol. 62, no. 5, pp. 2364-2373, 2011.

[13] A. Saadatmandi and M. Dehghan, "A new operational matrix for solving fractional-order differential equations," Computers \& Mathematics with Applications. An International Journal, vol. 59, no. 3, pp. 1326-1336, 2010.

[14] A. Saadatmandi and M. Dehghan, "The use of sinc-collocation method for solving multi-point boundary value problems," Communications in Nonlinear Science and Numerical Simulation, vol. 17, no. 2, pp. 593-601, 2012.

[15] R. E. Bellman and R. E. Kalaba, Quasilinearization and NonLinear Boundary Value Problems, Elsevier, New York, NY, USA, 1965.

[16] E. L. Stanley, Quasilinearization and Invariant Imbedding, Academic Press, New York, NY, USA, 1968.

[17] R. P. Agarwal and Y. M. Chow, "Iterative methods for a fourth order boundary value problem," Journal of Computational and Applied Mathematics, vol. 10, no. 2, pp. 203-217, 1984.

[18] C. A. Baird Jr., "Modified quasilinearization technique for the solution of boundary-value problems for ordinary differential equations," Journal of Optimization Theory and Applications, vol. 3, no. 4, pp. 227-242, 1969.

[19] V. B. Mandelzweig and F. Tabakin, "Quasilinearization approach to nonlinear problems in physics with application to nonlinear ODEs," Computer Physics Communications, vol. 141, no. 2, pp. 268-281, 2001.

[20] R. A. Khan and H. Khalil, "A new method based on legendre polynomials for solution of system of fractional order partial differential equations," International Journal of Computer Mathematics, vol. 91, no. 12, pp. 2554-2567, 2014.

[21] H. Khalil and R. A. Khan, "A new method based on Legendre polynomials for solutions of the fractional two-dimensional heat conduction equation," Computers \& Mathematics with Applications, vol. 67, no. 10, pp. 1938-1953, 2014.

[22] H. Khalil and R. A. Khan, "The use of Jacobi polynomials in the numerical solution of coupled system of fractional differential equations," International Journal of Computer Mathematics, vol. 92, no. 7, pp. 1452-1472, 2015.

[23] H. Khalil, R. A. Khan, D. Baleanu, and M. M. Rashidi, "Some new operational matrices and its application to fractional order Poisson equations with integral type boundary constrains," Computers \& Mathematics with Applications, 2016.

[24] H. Khalil, R. A. Khan, D. Baleanu, and S. H. Saker, "Approximate solution of linear and nonlinear fractional differential equations under m-point local and nonlocal boundary conditions," Advances in Difference Equations, vol. 2016, article 177, 2016.

[25] H. Khalil, M. M. Rashidi, and R. A. Khan, "Application of fractional order legendre polynomials: a new procedure for solution of linear and nonlinear fractional differential equations under m-point nonlocal boundary conditions," Communications in Numerical Analysis, vol. 6, no. 2, pp. 144-166, 2016.

[26] R. H. Bartels and G. W. Stewart, "Solution of the matrix equation $A X+X B=C[F 4]$," Communications of the ACM, vol. 15, no. 9, pp. 820-826, 1972.

[27] U. Saeed and M. ur Rehman, "Wavelet-Galerkin quasilinearization method for nonlinear boundary value problems," Abstract and Applied Analysis, vol. 2014, Article ID 868934, 10 pages, 2014.

[28] M. Rehman and R. A. Khan, "A numerical method for solving boundary value problems for fractional differential equations," Applied Mathematical Modelling, vol. 36, no. 3, pp. 894-907, 2012.

[29] X. Li and B. Wu, "Approximate analytical solutions of nonlocal fractional boundary value problems," Applied Mathematical Modelling, vol. 39, no. 5-6, pp. 1717-1724, 2015.

[30] F. Z. Geng and M. G. Cui, "A reproducing kernel method for solving nonlocal fractional boundary value problems," Applied Mathematics Letters, vol. 25, no. 5, pp. 818-823, 2012.

[31] R. Rach, J.-S. Duan, and A.-M. Wazwaz, "Solving coupled Lane-Emden boundary value problems in catalytic diffusion reactions by the Adomian decomposition method," Journal of Mathematical Chemistry, vol. 52, no. 1, pp. 255-267, 2014. 


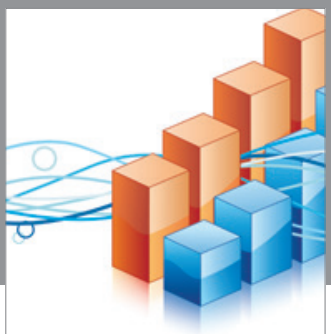

Advances in

Operations Research

vatem alat4

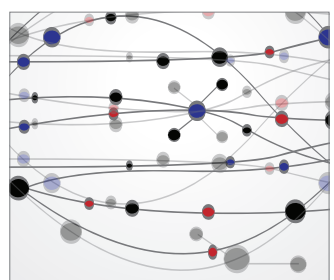

\section{The Scientific} World Journal
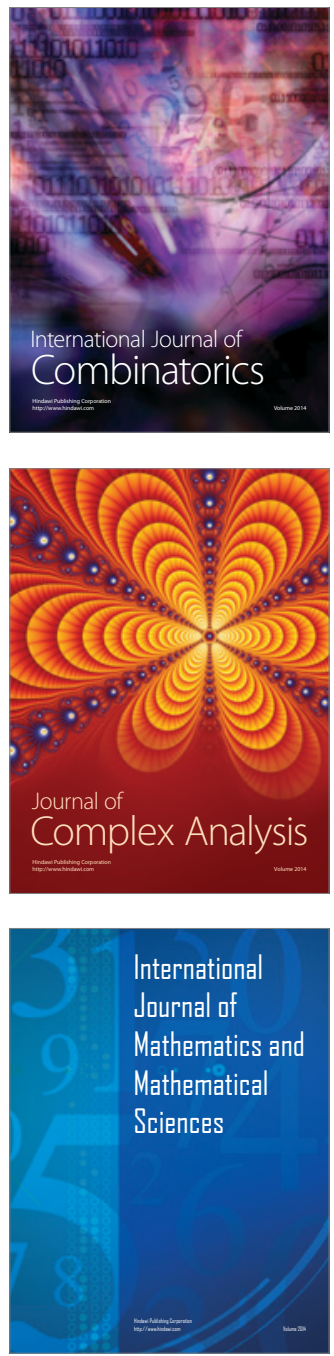
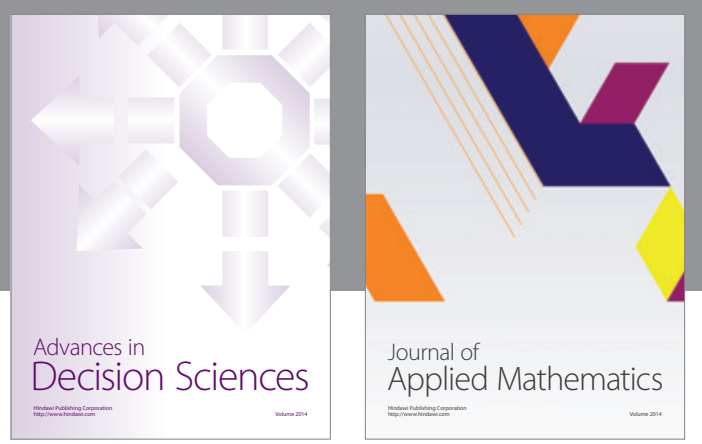

Algebra

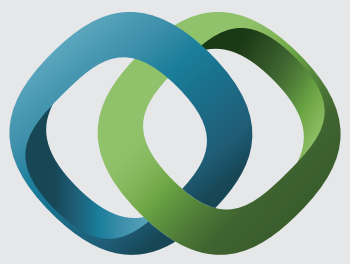

\section{Hindawi}

Submit your manuscripts at

http://www.hindawi.com
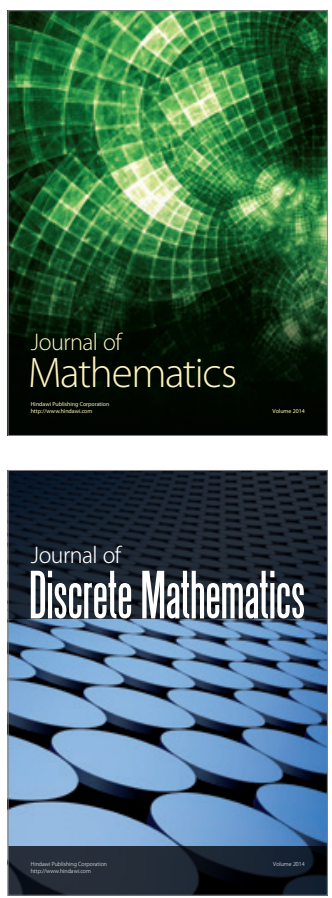

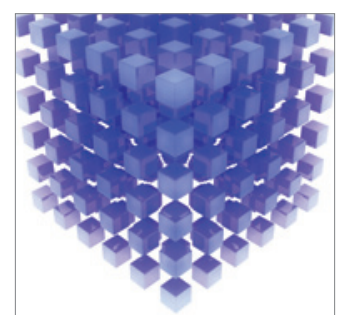

Mathematical Problems in Engineering
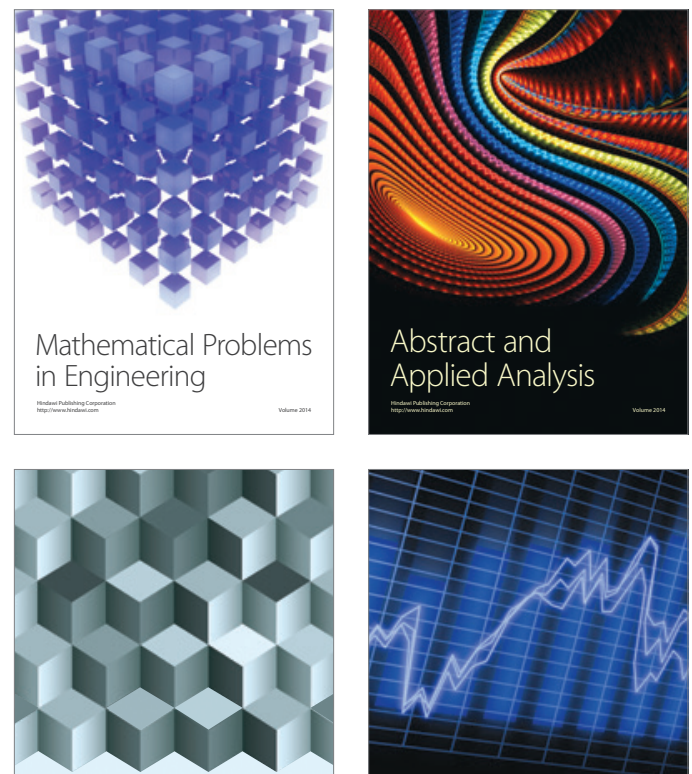

Journal of

Function Spaces

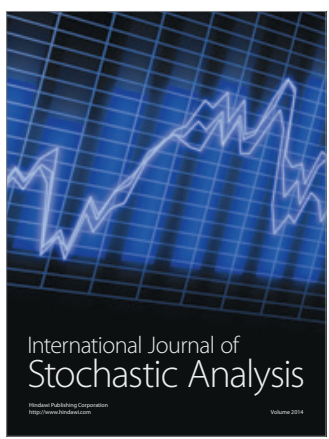

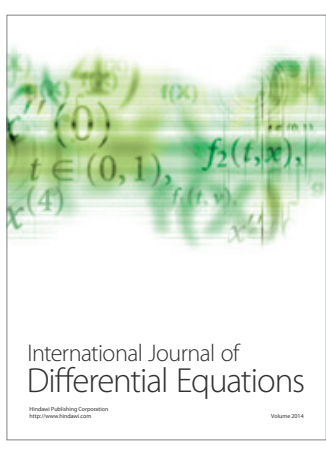
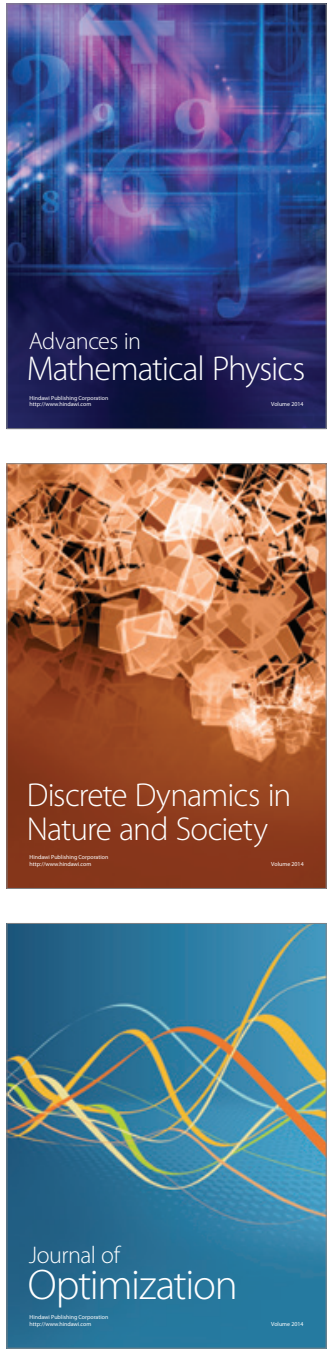